\title{
Demystifying the Formation of Colloidal Perovskite Nanocrystals Via Controlling Stepwise Synthesis
}

Aqiang Liu, Chenghao Bi, Xuanhui Qu, Jianjun Tian*

Beijing Advanced Innovation Center for Materials Genome Engineering, Institute for Advanced Materials and Technology, University of Science and Technology Beijing, 100083, China.

*Corresponding authors:

tianjianjun@mater.ustb.edu.cn 

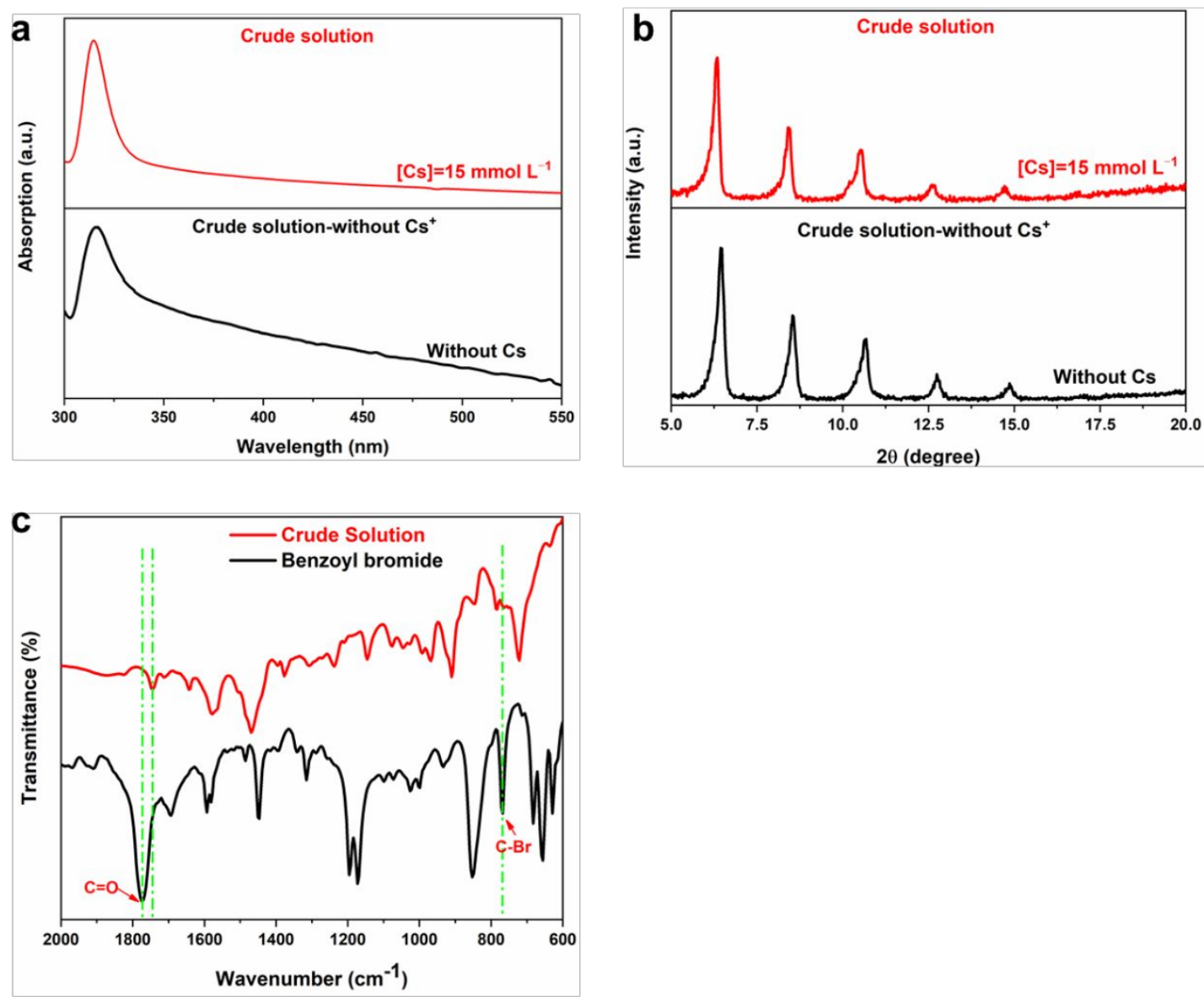

Figure S1. The characterization of the crude solutions and crude solution-without $\mathbf{C s}^{+}$ions. (a)The absorption spectra of the crude solutions and crude solution-without $\mathrm{Cs}^{+}$ ions. (b) The XRD pattern of the films made from the crude solution and crude solutionwithout $\mathrm{Cs}^{+}$ions; (c) The FTIR spectra of the benzoyl bromide and crude solution. 

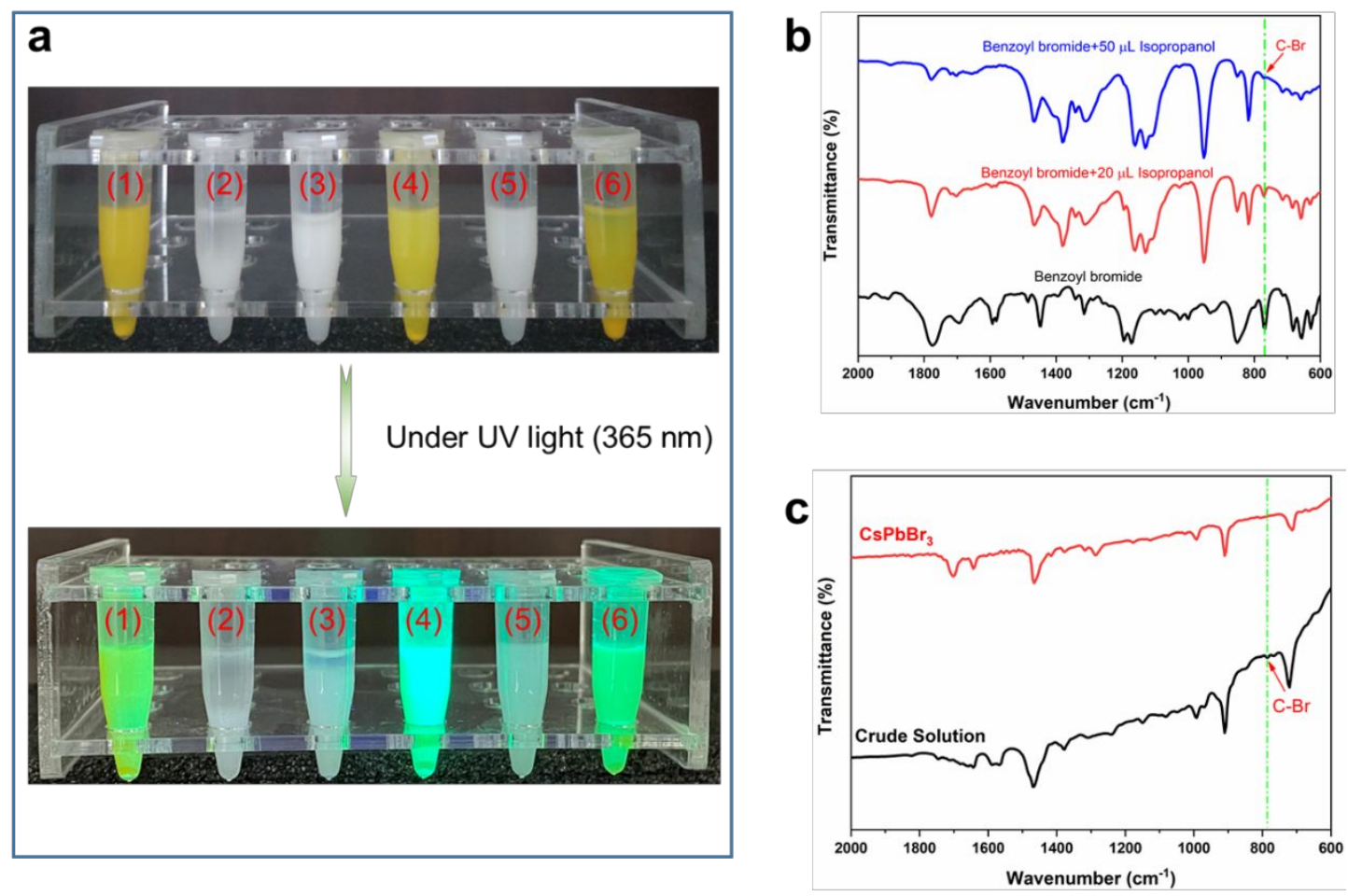

Figure S2. The study on the trigger mechanism of isopropanol. (a) The effect of adding different trigger agent to the crude solution: (1) methanol, (2) acetonitrile, (3) acetone, (4) isopropanol, (5) ethyl acetate, and (6) n-butanol. (b) The FTIR spectra of benzoyl bromide w/o addition of isopropanol. (c) The FTIR spectra of crude solution w/o addition of isopropanol. 

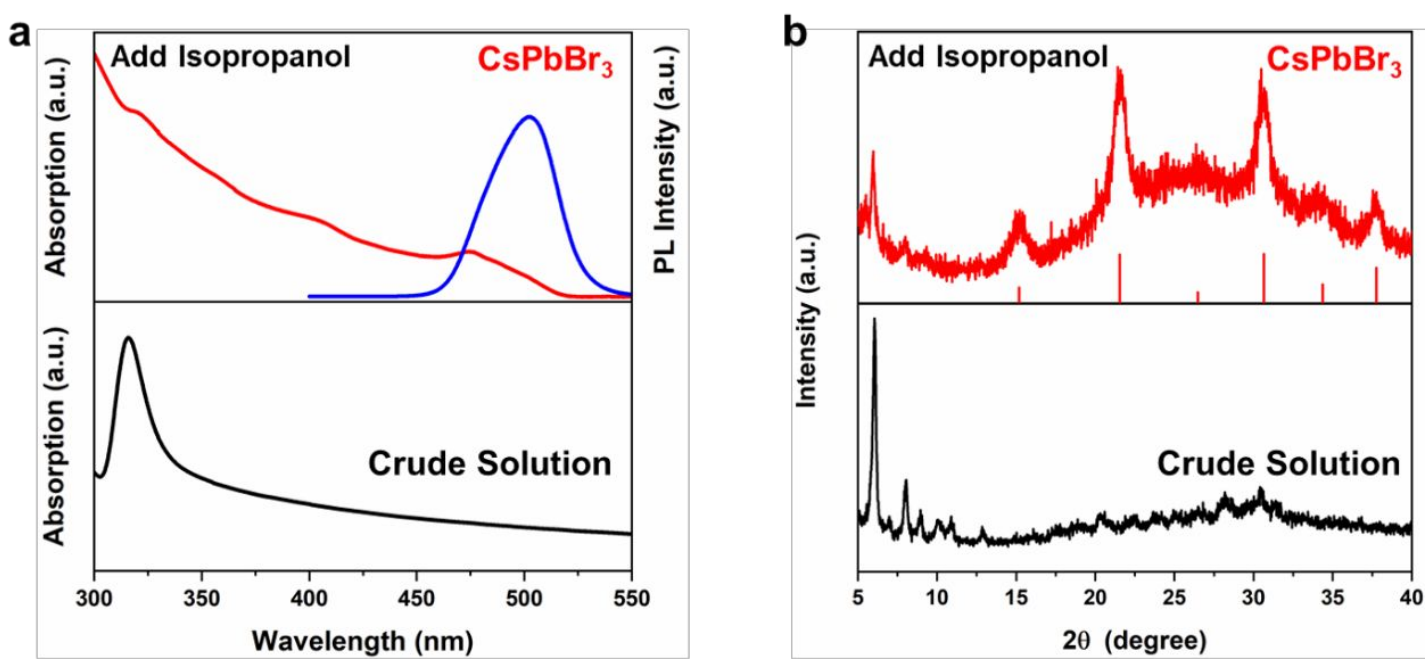

Figure S3. The PeNCs was synthesized by stepwise method using acetyl bromide as bromine source. (a) The absorption spectra and PL spectra of crude solution and PeNCs. (b) The XRD pattern of the films made from the crude solution w/o addition of isopropanol. 